\title{
ProfesiOnAL DE INSTRUMENTACIÓN QUIRÚRGICA FRENTE A LA APLICACIÓN DE LOS PRINCIPIOS Y VALORES BIOÉTICOS DE ACUERDO CON LA LEY DE TALENTO HUMANO EN SALUD EN COLOMBIA THE SURGICAL INSTRUMENTATION PROFESSIONAL IN LIGHT OF THE APPLICATION OF BIOETHICAL PRINCIPLES AND VALUES ACCORDING TO THE LAW ON HUMAN TALENT IN HEALTH IN COLOMBIA PROFISSIONAL DE INSTRUMENTAÇÃO CIRÚRGICA ANTE A APLICAÇÃO DOS PRINCÍPIOS E VALORES BIOÉTICOS DE ACORDO COM A LEI DE TALENTO HUMANO EM SAÚDE NA COLÔMBIA
}

Stephanye Carrillo-González* Jaime Lorduy-Gómez ${ }^{* *}$ Ruby Muñoz-Baldiris***

\begin{abstract}
RESUMEN
El instrumentador quirúrgico es un miembro activo del equipo quirúrgico, que tiene como esencia y objeto formal del ejercicio mantener la vida con todo el respeto y la dignidad inherentes al ser humano, y debe acompañar sus acciones de forma coherente a fin de proteger y preservar la vida, de esta manera le otorga significado a la enfermedad, el sufrimiento y el dolor. El objetivo del presente estudio descriptivo, de corte transversal y prospectivo, es determinar el conocimiento del instrumentador quirúrgico frente a los principios y valores bioéticos según la Ley 1164 de 2007. La población objeto fueron instrumentadores quirúrgicos de la
\end{abstract}

DOI: 10.5294/PEBI.2018.22.1.10

PARA CITAR ESTE ARTíCULO / TO REFERENCE THIS ARTICLE / PARA CITAR ESTE ARTIGO

Carrillo-González S, Lorduy-Gómez J, Muñoz-Baldiris R. Profesional de Instrumentación Quirúrgica frente a la aplicación de los Principios y Valores Bioéticos de acuerdo con la Ley de Talento Humano en Salud en Colombia. pers.bioét. 2018; 22(1): 134-147. DOI: 10.5294/pebi.2018.22.1.10

* $\quad$ orcid.org/0000-0001-8056-3361. Corporación Universitaria Rafael Nuñez, Colombia. stephanye.carrillo@curnvirtual.edu.co

** orcid.org/0000-0001-8941-5592. Corporación Universitaria Rafael Nuñez, Colombia. jaime.lorduy@curnvirtual.edu.co

*** orcid.org/0000-0002-4859-3170. Corporación Universitaria Rafael Nuñez, Colombia.ruby.munoz@curnvirtual.edu.co
FECHA DE RECEPCIÓN: 2017-01-11

FECHA DE ENVÍO A PARES: 2017-03-27

FECHA DE APROBACIÓN POR PARES: 2017-07-28

FECHA DE ACEPTACIÓN: 2018-02-22 
ciudad de Cartagena. Participaron profesionales de tres instituciones de salud. Se encontró que los principios éticos y bioéticos que conocen y aplican estos profesionales son: veracidad, igualdad y beneficencia. Los que menos conocen son mal menor, no maleficencia, de totalidad y de causa de doble efecto. Los valores más aplicados son: humanidad, dignidad, responsabilidad, prudencia y secreto. Se concluye que la gran mayoría de los instrumentadores quirúrgicos encuestados aplican los principios y valores éticos en su quehacer profesional, pero desconocen el nombre o la definición de los mismos. Se propone ahondar más desde la academia en el fortalecimiento de estos temas para lograr un nivel integral y de mayor cuidado.

Palabras Clave: principios morales; valores sociales; humanización de la atención; paciente; educación; ética; bioética (Fuente: DeCS).

\section{Abstract}

A surgical instrumentation professional is an active member of the surgical team whose essence and formal objective is to maintain life with all the respect and dignity inherent in the human being. The actions of these professionals must be accompanied in a consistent way, so as to protect and preserve life, and thus give meaning to illness, suffering and pain. The objective of this descriptive and cross-sectional study of a prospective nature is to determine what the surgical instrumentation professional knows about bioethical principles and values in light of Colombian Law 1164/ 2007. The target population included surgical instrumentation professionals in the city of Cartagena, specifically, from three health institutions. It was found that veracity, equality and beneficence are the ethical and bioethical principles these professionals know and apply. Those known the least are lesser evil, doing no harm, totality and the principle of the cause of double effect. The values applied the most are: humanity, dignity, responsibility, prudence and confidentiality. It was concluded that the vast majority of surgical instrumentation professionals who were surveyed apply ethical principles and values in their professional practice, but they are unable to name them or to define what they mean. It is suggested the academic community do more to delve into these issues in order to strengthen them for the sake of achieving a comprehensive level of care.

Keywords: Moral principles; social values; humanization of care; patient; education; ethics; bioethics (Source: DeCS).

\section{Resumo}

O instrumentador cirúrgico é um membro ativo da equipe cirúrgica, que tem como essência e objeto formal do exercício manter a vida com todo o respeito e a dignidade inerentes ao ser humano, e deve acompanhar suas ações de forma coerente a fim de proteger e preservar a vida; dessa maneira, o profissional outorga significado à doença, ao sofrimento e à dor. O objetivo do presente estudo descritivo, de corte transversal e prospectivo, é determinar o conhecimento do instrumentador cirúrgico diante dos princípios e valores bioéticos de acordo com a Lei 1.164 de 2007. A população-alvo foram instrumentadores cirúrgicos da cidade de Cartagena. Participaram profissionais de três instituições de saúde. Descobriu-se que os princípios éticos e bioéticos que esses profissionais conhecem e aplicam são: veracidade, igualdade e beneficência. Os princípios que eles menos conhecem são mal menor, não maleficência, de totalidade e de causa com duplo efeito. Os valores mais aplicados são: humanidade, dignidade, responsabilidade, prudência e segredo. Conclui-se que a grande maioria dos instrumentadores cirúrgicos entrevistados aplicam os princípios e valores éticos em sua atividade profissional, mas desconhecem o nome ou a definição deles. Propõe-se aprofundar mais, a partir da academia, no fortalecimento desses temas para alcançar um nível integral e de maior cuidado.

Palavras Chave: bioética; educação; ética; humanização do atendimento; paciente; princípios morais; valores sociais (Fonte: DeCS). 


\section{INTRODUCCIÓN}

Desde los inicios de la humanidad se conoce la complejidad de los seres humanos y las diferencias que tienen en sus formas de sentir, pensar e interactuar con los demás. Dentro de las características del ser humano está el hecho de ser sociable; los filósofos de todos los tiempos han concluido que el hombre es un ser social por naturaleza o, en otras palabras, que la sociedad es, para el hombre, algo natural (1). Esta persona humana compleja es la que constituye la esencia de los profesionales de la salud y de los pacientes, quienes operan desde roles diferentes de necesidad. Dentro del equipo quirúrgico se encuentra el instrumentador quirúrgico, el cual cumple un papel muy importante, ya que este profesional de la salud tiene como esencia y objeto formal del ejercicio mantener la vida con todo el respeto y la dignidad inherentes al ser humano, y debe acompañar sus acciones de forma coherente, con el objetivo de proteger y preservar la vida, de esta manera le otorga significado a la enfermedad, el sufrimiento y el dolor. Sin embargo, por múltiples factores, el talento humano en salud ha entrado en el terreno de la deshumanización. Por ello es necesario estimular en el profesional de la salud, incluido el instrumentador quirúrgico, la vocación por el servicio, e integrar al saber científico la sensibilidad humana durante la formación; si tenemos esta primicia ganada, seremos capaces de lograr que los principios éticos y valores humanos que se brindan apoyen el compromiso personal, profesional e institucional que lleve a generar confianza y gratitud en el paciente, su familia y la comunidad; de este modo se logra prestigio y liderazgo social.

\section{El instrumentador quirúrgico y su compromiso con los principios y valores éticos y bioéticos}

A lo largo de los tiempos, el instrumentador quirúrgico se ha profesionalizado en su saber teniendo en cuenta el avance científico, y asume cada día el compromiso que posee en la atención de los pacientes quirúrgicos, perfeccionando sus técnicas y conocimientos para brindar al final una mejor atención al paciente. En general existe un enfoque que busca cuidar y velar por los protocolos que hay que cumplir, por ejemplo: las reglas asépticas que evitan una infección cruzada, y otras necesidades en el ambiente quirúrgico, las cuales nos convierten en profesionales con destreza y con mucha solidez científica.

Si bien es cierto que el instrumentador quirúrgico tiene varios perfiles ocupacionales en los que se ha destacado, es en el área asistencial en la que predomina su accionar, teniendo en cuenta que el ambiente hospitalario es uno de los más difíciles, por lo que es el área de quirófano donde los pacientes llegan con más necesidades afectivas, sumadas a las físicas propias de su estado. Para llevar a cabo estos fines, cada persona que interviene en los procedimientos propios del acto quirúrgico debe poseer 


\section{EL INSTRUMENTADOR QUIRÚRGICO, INDISPENSABLE POR SU FORMACIÓN ACADÉMICA INTEGRAL, CUMPLE FUNCIONES SIGNIFICATIVAS DENTRO DEL EQUIPO QUIRÚRGICO, ENTRE ELLAS: COORDINAR \\ LAS ÁREAS DE CIRUGÍA Y CENTRALES DE ESTERILIZACIÓN, PARTICIPAR ACTIVAMENTE EN EL ACTO QUIRÚRGICO, MANEJAR EQUIPOS BIOMÉDICOS, ENTRE OTRAS FUNCIONES.}

conocimientos y entrenamientos adecuados, adquiridos a través de una formación académica.

El instrumentador quirúrgico, indispensable por su formación académica integral, cumple funciones significativas dentro del equipo quirúrgico, entre ellas: coordinar las áreas de cirugía y centrales de esterilización, participar activamente en el acto quirúrgico, manejar equipos biomédicos, entre otras funciones.

Ahora bien, teniendo en cuenta el rol y la importancia en el ambiente hospitalario del instrumentador quirúrgico es pertinente preguntarse: ¿conoce este profesional la Ley 1164 de 2007 donde se registran los principios, valores, deberes y derechos que debe poseer el talento humano en salud aquí en Colombia? (2) ¿Aplica los principios y valores en los actos donde se intervenga a un paciente? En relación con estas preguntas no existen estudios previos en Colombia relativos a esta profesión, por ello se deriva la presente investigación.

\section{Principios y valores aplicados por el instrumentador quirúrgico}

Según la Ley 1164, capítulo VI, de 2007 (2), en Colombia existen unos principios y valores que rigen a todo el personal que interviene en la promoción, educación, información, prevención, diagnóstico, tratamiento, rehabilitación y paliación de la enfermedad de to- dos los habitantes del territorio nacional, dentro de la estructura organizacional de la prestación de los servicios de salud.

Para profundizar sobre el tema es importante partir del concepto de principio (del latín principŭum): "primer instante del ser de algo, punto que se considera como primero en una extensión o en una cosa, norma o idea fundamental que rige el pensamiento o la conducta” (3).

Principios: según la Ley de Talento Humano en Salud, artículo 35, estos se enmarcan en el contexto del cuidado respetuoso de la vida y la dignidad de cada ser humano, y en la promoción de su desarrollo existencial, procurando su integridad física, genética, funcional, psicológica, social, cultural y espiritual, sin distinciones de edad, credo, sexo, raza, nacionalidad, lengua, cultura, condición socioeconómica e ideología política, y de un medio ambiente sano (2).

A continuación se parafrasean los principios aplicados en el quehacer del instrumentador quirúrgico en aras de buscar aplicabilidad:

Veracidad. Orienta el desempeño del instrumentador quirúrgico desde una comunión entre lo que es, piensa, dice y hace, en referencia al trato con las personas en el ejercicio de su profesión. 
Autonomía. Es la capacidad que tiene el instrumentador quirúrgico para deliberar, decidir y actuar, con base en un conocimiento científico, a favor de los demás.

Igualdad. El instrumentador quirúrgico reconoce el mismo derecho para todos los que se benefician de su servicio de recibir una adecuada atención en salud, acorde con las necesidades de cada ser humano.

Beneficencia. En el ejercicio de su profesión, instrumentador quirúrgico debe hacer lo que conviene a cada ser humano respetando los derechos de los demás y procurando que el beneficio para el paciente sea más abundante y menos demandante de esfuerzos en términos de riesgos y costos. Debe respetar de modo especial los derechos de grupos vulnerables limitados en el ejercicio de su autonomía.

No maleficencia. Orienta el desempeño del instrumentador hacia actos que, aunque no beneficien, puedan evitar daño, tratando siempre de defender los mejores intereses del paciente.

Mal menor. En el ejercicio de la profesión, ante una situación donde hay que actuar sin dilación, el instrumentador quirúrgico deberá elegir el menor mal, evitando transgredir el derecho a la integridad y las posibles consecuencias que se deriven de no actuar.

De totalidad. En el ejercicio profesional el instrumentador quirúrgico participa en actos donde se hace necesaria la extirpación de una parte del cuerpo que cause daño al organismo, siempre y cuando se apliquen las condiciones para la supresión del miembro y en beneficio de la conservación total del individuo.
De doble efecto. Es éticamente aceptable participar en actos donde la acción en sí misma sea buena o indiferente, y tenga un doble efecto (uno bueno y uno malo), siempre y cuando: el efecto bueno sea inmediato, el fin de quien realiza la acción sea honesto, y exista una causa proporcionada para permitir el efecto malo.

Valores: El ejercicio de la profesión de Instrumentación Quirúrgica se realizará teniendo en cuenta los siguientes valores brindados por el artículo 36 de la Ley 1164 de 2007, aplicados a las otras personas, la comunidad, la profesión y las instituciones. Sirven de fundamento a las disposiciones sobre ética profesional del instrumentador quirúrgico y son los siguientes:

Humanidad. El instrumentador quirúrgico debe dar un trato humano a cada paciente de acuerdo con una jerarquía razonablemente sustentada de sus necesidades biológicas, psicológicas, sociales y espirituales.

Dignidad. El profesional de instrumentación quirúrgica debe reconocer la dignidad de cada ser humano, entendida como la mayor excelencia entre los seres vivos, por lo que en su actuar no participa en ninguna forma de maltrato o discriminación, antes bien, promueve al ser humano dentro de las características especiales, tratándolo como fin, nunca como un medio.

Responsabilidad. Se debe analizar, dar razón y asumir las consecuencias de las propias acciones u omisiones, en lo referente al ejercicio de la profesión.

Prudencia. El instrumentador quirúrgico debe tener sensatez en la conducta práctica; debe realizar una valoración acertada de los medios y de los fines de cada acto; ponderar previamente el fin que desea alcanzar, 


\section{EL INSTRUMENTADOR QUIRÚRGICO DEBE}

SALVAGUARDAR LA CONFIDENCIALIDAD

EN TODO LO QUE VIERE U OYERE EN EL

ÁMBITO DONDE SE DESENVUELVE; DE IGUAL

FORMA, DEBE EVIDENCIAR CONFIABILIDAD Y

CREDIBILIDAD EN EL CUMPLIMIENTO DE LOS

COMPROMISOS QUE ADQUIERE EN EL EJERCICIO

DE SU PROFESIÓN.

cuáles son sus consecuencias positivas y negativas para sí mismo, para los pacientes, el equipo de trabajo, y cuáles son los medios y el momento más adecuado para alcanzarlo.

Secreto: el instrumentador quirúrgico debe salvaguardar la confidencialidad en todo lo que viere u oyere en el ámbito donde se desenvuelve; de igual forma, debe evidenciar confiabilidad y credibilidad en el cumplimiento de los compromisos que adquiere en el ejercicio de su profesión.

\section{MATERIALES Y MÉTODOS}

Estudio descriptivo, de corte transversal y prospectivo. La población objeto fueron instrumentadores quirúrgicos que se encontraban laborando en la ciudad de Cartagena y que tuvieran contacto con estudiantes en formación, especialmente en las áreas asistencial, administrativa, comercial y de docencia. Participaron profesionales de tres instituciones de salud de acuerdo con los criterios de inclusión (22 instrumentadores quirúrgicos entre las tres IPS).
La fuente de información de la investigación es primaria. Se diseñó un instrumento tipo cuestionario basado en situaciones que enfrentaría el instrumentador quirúrgico en su ejercicio profesional, en el que se preguntó sobre el conocimiento de los principios y valores de la Ley de Talento Humano en Salud de Colombia.

Teniendo en cuenta la información recolectada se creó una base de datos en el programa excel, los cuales se categorizarán según la naturaleza de las variables. Para una mejor tabulación y análisis de la información, esta base de datos se exportó al programa estadístico SPSS V22. Los cálculos se realizaron de acuerdo con la naturaleza de las variables, y la información de las variables cualitativas se organizó en tablas de frecuencia y porcentajes. Las variables cuantitativas se agruparon en categorías a fin de realizar un mejor análisis de la información.

En relación con las consideraciones éticas, para este estudio se tuvieron en cuenta: a nivel internacional, el Código de Núremberg (4), la Declaración de Helsinki (5) y el Informe Belmont (6), y la reglamentación colombiana vigente, entre otras, la Resolución 008430 de 1993). Por la misma naturaleza de la investigación y la manera como se recogieron los datos se clasifica como una investigación sin riesgos (7). Cabe aclarar que la información fue suministrada bajo consentimiento de los instrumentadores quirúrgicos encuestados, y no se tiene en cuenta información que comprometa la identidad de los mismos.

\section{RESULTADOS}

Al comparar los principios de la bioética aplicados al talento humano en salud en Colombia, de acuerdo con la Ley 1164 de 2007 (Ley de Talento Humano en 
Salud), capítulo VI, y la percepción del instrumentador quirúrgico para evidenciarlos encontramos lo siguiente: en las características sociodemográficas se observa que existe comparabilidad entre los diferentes grupos de edad de la población. Con relación al nivel de formación presentan una formación de pregrado $(\mathrm{n}=16)$, lo que representa $72 \%$. Con relación al área de desempeño se destaca el área asistencial $(\mathrm{n}=17)$ con un $77,3 \%$. De igual forma, el 59,1\% ( $\mathrm{n}=13)$ tiene más de dos años de experiencia. Finalmente, respecto a la religión, 72,7\% $(\mathrm{n}=16)$ profesan la religión católica (tabla 1).

Tabla 1. Características sociodemográficas de profesionales de Instrumentación Quirúrgica frente a los principios y valores bioéticos de acuerdo con la Ley de Talento Humano en Salud de Colombia

\begin{tabular}{|c|c|c|c|}
\hline Variables sociodemográficas & Categorías & $\mathbf{N}$ & $\%$ \\
\hline \multirow{4}{*}{ Edad } & Entre 22 y 24 años & 6 & 27,3 \\
\hline & Entre 25 y 31 años & 5 & 22,7 \\
\hline & Entre 32 y 36 años & 6 & 27,3 \\
\hline & Entre 37 y 46 años & 5 & 22,7 \\
\hline \multirow{3}{*}{ Nivel de formación } & Pregrado & 16 & 72,7 \\
\hline & Especialista & 4 & 18,2 \\
\hline & Maestría & 2 & 9,1 \\
\hline \multirow{4}{*}{ Área de desempeño } & Asistencial & 17 & 77,3 \\
\hline & Administrativa & 3 & 13,6 \\
\hline & Comercial & 1 & 4,5 \\
\hline & Docente & 1 & 4,5 \\
\hline \multirow{2}{*}{$\begin{array}{l}\text { Más de dos años de } \\
\text { experiencia }\end{array}$} & Sí & 13 & 59,1 \\
\hline & No & 9 & 40,9 \\
\hline \multirow{3}{*}{ Confesión religiosa } & Católica & 16 & 72,7 \\
\hline & Cristiana & 5 & 22,7 \\
\hline & Otra & 1 & 4,5 \\
\hline
\end{tabular}

Fuente: datos obtenidos por los investigadores.
De acuerdo con la respuesta de los encuestados ante las situaciones donde aplicarían los principios éticos según la Ley de Talento Humano en relación con su ejercicio profesional y el trato con los compañeros, los instrumentadores actuarían de manera correcta conforme a lo que piensan frente a una situación en el ámbito quirúrgico aunque eso afecte su relaciones personales $(\mathrm{n}=17)$, lo que representa el $77,3 \%$; sin embargo, es interesante anotar que un $22,7 \%$, aunque piensan en actuar correctamente, no lo harían solo por no dañar su relación con los demás compañeros.

La mayoría de los instrumentadores encuestados admitió que había cometido un error $(\mathrm{n}=16)$, lo que representa el $72,7 \%$. Estos manifestaron los errores cometidos ante los entes encargados con el fin de beneficiar al paciente $(\mathrm{n}=13)$, lo que representa el $59,1 \%$.

Es interesante notar que respecto a la realización de procedimientos que atentan contra la dignidad de los pacientes un $50 \%$ estuvo de acuerdo y un $50 \%$ en desacuerdo.

Del porcentaje de los instrumentadores quirúrgicos que no están de acuerdo con un procedimiento que atente contra la dignidad del paciente, la respuesta del equipo quirúrgico ante esto es que: un $22,7 \%$ no entienden al instrumentador quirúrgico, un 13,6\% lo entiende y un 9,1\% rechaza su posición.

Los instrumentadores consultados en el presente estudio, al enfrentarse a una decisión de realizar un procedimiento prioritario a un paciente que por su estado urgente y vulnerable necesite atención incluso antes que otro previamente programado, respondieron que lo harían en un 90,9\% ( $\mathrm{n}=20)$; sin embargo, es interesante anotar que un $9,1 \%$ manifestó que no $(\mathrm{n}=2)$. 
Finalmente, ante la disyuntiva de si, como parte del equipo quirúrgico, saben que la utilización de un elemento o insumo en el paciente conlleva consecuencias inmediatas buenas, pero se observan efectos negativos a largo plazo, un 95,5\% reportaría la situación $(n=21)$. Sin embargo, un 4,5\% ( $\mathrm{n}=1)$ seguiría usando el elemento o insumo para evitar problemas con las directivas de la empresa donde labora (tabla 2).

De acuerdo con la respuesta de los encuestados ante las situaciones donde aplicarían los valores éticos según el capítulo VI de la Ley de Talento Humano, un 95,5\% $(\mathrm{n}=21)$ piensa que se debería dar un apoyo al paciente en los aspectos psicológicos y espirituales, y no solo limitarse al aspecto biológico, y consideran que esto hace parte de su trabajo.

La opinión de los encuestados en cuanto al respeto de las creencias de los pacientes antes de realizar un procedimiento es que un $77,3 \%(n=17)$ siempre lo hace, pero interesante que en situaciones especiales algunos las ignorarían ( $\mathrm{n}=5)$, lo que representa un $22,7 \%$.

En relación con la intervención del instrumentador quirúrgico cuando observa que un paciente es maltratado, discriminado o instrumentalizado, un 90,9\% $(\mathrm{n}=20)$ respondió que intervendría; sin embargo, llama la atención que un pequeño grupo no lo haría $(\mathrm{n}=2)$, lo que representa un $9,1 \%$.

En otra respuesta se evidencia que un $95,5 \%$ reporta los eventos adversos que existieron en el acto quirúrgico. Ante la petición de reserva por la ocurrencia de un evento donde se compromete la privacidad del paciente, un $81,8 \%(\mathrm{n}=10)$ respondió que guardaría el secreto (tabla 3$)$.
En cuanto al conocimiento acerca de los principios éticos y bioéticos de la Ley 1164 de 2007, capítulo VI, un $68,2 \%$ de los instrumentadores quirúrgicos refirió conocerla, y el $27,3 \%$ no la conoce. Por otra parte, un $22,7 \%$ de los encuestados conoce de los aspectos éticos y bioéticos por sus propios medios o por un amigo, un $18,2 \%$ por una capacitación laboral y un $9,1 \%$ por los estudios de posgrado.

Específicamente, los principios éticos y bioéticos que conocen los instrumentadores quirúrgicos son: veracidad, $54,5 \%(\mathrm{n}=12)$; igualdad, $72,7 \%(\mathrm{n}=16)$; beneficencia, $45,5 \%(\mathrm{n}=12)$. Los que menos conocen son: mal menor $73 \%(\mathrm{n}=16)$; no maleficencia $63,6 \%(\mathrm{n}=14)$; totalidad y causa de doble efecto $77,3 \%(\mathrm{n}=17)$. El principio de autonomía lo conocen el $50 \%$ de los instrumentadores quirúrgicos encuestados, sin embargo, en esa misma medida un $50 \%$ lo desconoce $(\mathrm{n}=11)($ tabla 4$)$.

En relación con el conocimiento de los valores éticos y bioéticos se evidencia lo siguiente: humanidad, $81,8 \%$ $(\mathrm{n}=18)$; dignidad, 63,6\% $(\mathrm{n}=14)$; responsabilidad $72,7 \%(\mathrm{n}=16)$; prudencia, 68,2\% $(\mathrm{n}=15)$; secreto, $63,6 \%(\mathrm{n}=14)$.

\section{DISCUSIÓN}

En este estudio se realiza una aproximación a lo que se evidencia en el día a día del instrumentador quirúrgico como un profesional de la salud.

En relación a investigaciones previas en el tema:

Según el análisis realizado en el estudio de caracterización del Instrumentador Quirúrgico en Colombia, respecto a la misión de formación, 
Tabla 2. Características relacionadas con situaciones donde se observa la aplicación de los principios de profesionales de Instrumentación Quirúrgica de acuerdo con la Ley de Talento Humano en Salud de Colombia

\begin{tabular}{|c|c|c|c|}
\hline Variables & Categorías & $\mathbf{N}$ & $\%$ \\
\hline \multirow[t]{2}{*}{ En relación con el ejercicio profesional y el trato con los compañeros } & $\begin{array}{l}\text { Pensar de manera correcta pero no actuar conforme a } \\
\text { esa forma de pensar }\end{array}$ & 5 & 22,7 \\
\hline & Actuar de manera correcta conforme a lo que pienso & 17 & 77,3 \\
\hline \multirow{2}{*}{ ¿Ha cometido errores en el ejercicio de su profesión? } & Sí & 16 & 72,7 \\
\hline & No & 6 & 27,3 \\
\hline \multirow{3}{*}{$\begin{array}{l}\text { Todos los errores cometidos fueron reportados conforme a los } \\
\text { procedimientos establecidos }\end{array}$} & Sí & 13 & 59,1 \\
\hline & No & 2 & 9,1 \\
\hline & No aplica & 7 & 31,8 \\
\hline \multirow{2}{*}{$\begin{array}{l}\text { ¿Alguna vez ha tenido preferencias por un paciente en el ejercicio de su } \\
\text { profesión? }\end{array}$} & Sí & 8 & 36,4 \\
\hline & No & 14 & 63,6 \\
\hline \multirow{5}{*}{ ¿En qué circunstancia tuvo el trato preferencial por esa persona? } & No aplica & 15 & 68,2 \\
\hline & Familiar o conocido & 2 & 9,1 \\
\hline & Niños & 3 & 13,6 \\
\hline & Urgencias & 1 & 4,5 \\
\hline & Pacientes terminales & 1 & 4,5 \\
\hline \multirow{2}{*}{$\begin{array}{l}\text { ¿En alguna situación de su ejercicio profesional ha estado en desacuerdo con } \\
\text { la realización de un procedimiento en un paciente porque atenta contra la } \\
\text { dignidad de este? }\end{array}$} & Sí & 11 & 50,0 \\
\hline & No & 11 & 50,0 \\
\hline \multirow{4}{*}{$\begin{array}{l}\text { Al estar en desacuerdo con la realización de un procedimiento en un } \\
\text { paciente, ¿cuál ha sido la respuesta del equipo quirúrgico? }\end{array}$} & Lo entendieron & 3 & 13,6 \\
\hline & No lo entendieron & 5 & 22,7 \\
\hline & Fue rechazado & 2 & 9,1 \\
\hline & No aplica & 12 & 54,5 \\
\hline \multirow{2}{*}{$\begin{array}{c}\text { ¿Participa usted en la decisión de realizar un procedimiento prioritario a un } \\
\text { paciente que por su estado urgente y vulnerable necesite atención incluso } \\
\text { antes que otro previamente programado? }\end{array}$} & Sí & 20 & 90,9 \\
\hline & No & 2 & 9,1 \\
\hline \multirow{2}{*}{$\begin{array}{l}\text { ¿En una situación de urgencias rompería un principio de asepsia que podría } \\
\text { acarrear una infección del sitio operatorio para evitar la muerte del paciente? }\end{array}$} & Sí & 16 & 72,7 \\
\hline & No & 6 & 27,3 \\
\hline \multirow{2}{*}{$\begin{array}{c}¿ \text { ¿En su ejercicio profesional consideraría no participar en un procedimiento } \\
\text { en que su presencia (por enfermedad física o emocional) implique el } \\
\text { deterioro de la salud del paciente? }\end{array}$} & Decido participar & 5 & 22,7 \\
\hline & No decido participar & 17 & 77,3 \\
\hline \multirow{2}{*}{$\begin{array}{c}\text { ¿Que piensa si en una cirugía, como parte del equipo quirúrgico, conoce que } \\
\text { las consecuencias inmediatas de la utilización de un elemento o insumo en el } \\
\text { paciente son buenas, pero se observan efectos negativos a largo plazo? }\end{array}$} & Lo seguiría usando para evitar problemas & 1 & 4,5 \\
\hline & Reporto la situación & 21 & 95,5 \\
\hline
\end{tabular}

Fuente: datos obtenidos por los investigadores. 
Tabla 3. Características relacionadas con situaciones donde se observa la aplicación de los valores de profesionales de Instrumentación Quirúrgica de acuerdo con la Ley de Talento Humano en Salud de Colombia

\begin{tabular}{|c|c|c|c|}
\hline Variables & Categorías & $\mathbf{N}$ & $\%$ \\
\hline \multirow{2}{*}{$\begin{array}{l}\text { ¿Piensa que como instrumentador quirúrgico debería dar un apoyo al paciente } \\
\text { en los aspectos psicológicos, espirituales y no solo limitarse al aspecto biológico? }\end{array}$} & También hace parte de mi trabajo & 21 & 95,5 \\
\hline & Eso no hace parte de mi trabajo & 1 & 4,5 \\
\hline \multirow{2}{*}{$\begin{array}{l}\text { ¿En la institución donde labora respeta usted las creencias de los pacientes en } \\
\text { relación con el procedimiento por realizar? }\end{array}$} & Siempre & 17 & 77,3 \\
\hline & En situaciones especiales las ignoraría & 5 & 22,7 \\
\hline \multirow{2}{*}{$\begin{array}{l}\text { ¿Intervendría alguna vez como instrumentador quirúrgico a favor del paciente } \\
\text { si observa que este es maltratado, discriminado o instrumentalizado? }\end{array}$} & Interviene & 20 & 90,9 \\
\hline & No interviene & 2 & 9,1 \\
\hline \multirow{2}{*}{$\begin{array}{l}\text { ¿En el ámbito laboral los pacientes tienen facilidad de acceso a usted como } \\
\text { profesional? }\end{array}$} & Sí & 18 & 81,8 \\
\hline & No & 4 & 18,2 \\
\hline \multirow{2}{*}{$\begin{array}{l}\text { ¿Reporta los eventos adversos que se tuvieron en el acto quirúrgico con el fin } \\
\text { de beneficiar al paciente? }\end{array}$} & Sí & 21 & 95,5 \\
\hline & No & 1 & 4,5 \\
\hline \multirow{3}{*}{$\begin{array}{l}\text { ¿Tiene usted como instrumentador libertad antes de un acto quirúrgico para } \\
\text { ponderar previamente el fin que desea alcanzar, las consecuencias positivas y } \\
\text { negativas para sí mismo, para los pacientes y el equipo de trabajo? }\end{array}$} & Siempre & 8 & 36,4 \\
\hline & A veces & 12 & 54,5 \\
\hline & Nunca & 2 & 9,1 \\
\hline \multirow{2}{*}{$\begin{array}{l}\text { ¿Ha estado en situaciones donde se ha visto en la necesidad de usar insumos no } \\
\text { apropiados para un paciente por no tener otras opciones en la empresa? }\end{array}$} & Sí, pero he estado en desacuerdo & 10 & 45,5 \\
\hline & Nunca he vivido esa situación & 12 & 54,5 \\
\hline \multirow{3}{*}{$\begin{array}{l}\text { En el momento de la cirugía, si observa un evento donde se compromete la } \\
\text { privacidad del paciente y le piden reserva ante el mismo ¿cuál es su actuación? }\end{array}$} & Guarda el secreto & 18 & 81,8 \\
\hline & No guarda el secreto & 1 & 4,5 \\
\hline & No sabe, no responde & 3 & 13,6 \\
\hline
\end{tabular}

Fuente: datos obtenidos por los investigadores.

Tabla 4. Conocimiento de los principios en profesionales de Instrumentación Quirúrgica de acuerdo con la Ley de Talento Humano en Salud de Colombia

\begin{tabular}{|c|c|c|c|c|c|c|c|c|c|c|c|c|c|c|c|c|}
\hline \multirow{2}{*}{$\begin{array}{c}\text { Variables } \\
\text { Categorías }\end{array}$} & \multicolumn{2}{|c|}{ Veracidad } & \multicolumn{2}{|c|}{ Igualdad } & \multicolumn{2}{|c|}{ Autonomía } & \multicolumn{2}{|c|}{ Beneficencia } & \multicolumn{2}{|c|}{ Mal menor } & \multicolumn{2}{|c|}{ No maleficencia } & \multicolumn{2}{|c|}{ Totalidad } & \multicolumn{2}{|c|}{ Doble efecto } \\
\hline & $\mathbf{N}$ & $\%$ & $\mathbf{N}$ & $\%$ & $\mathbf{N}$ & $\%$ & $\mathbf{N}$ & $\%$ & $\mathbf{N}$ & $\%$ & $\mathbf{N}$ & $\%$ & $\mathbf{N}$ & $\%$ & $\mathbf{N}$ & $\%$ \\
\hline Sí & 12 & 54,5 & 16 & 72,7 & 11 & 50 & 12 & 54,5 & 6 & 27 & 8 & 36,4 & 5 & 22,7 & 5 & 22,7 \\
\hline No & 10 & 45,5 & 6 & 27,3 & 11 & 50 & 10 & 45,5 & 16 & 73 & 14 & 63,6 & 17 & 77,3 & 17 & 77,3 \\
\hline
\end{tabular}

Fuente: datos obtenidos por los investigadores. 
Tabla 5. Conocimiento de los valores en profesionales de Instrumentación Quirúrgica de acuerdo con la Ley de Talento Humano en Salud de Colombia

\begin{tabular}{|c|c|c|c|c|c|c|c|c|c|c|}
\hline Variables & \multicolumn{2}{|c|}{ Humanidad } & \multicolumn{2}{|c|}{ Dignidad } & \multicolumn{2}{c|}{ Responsabilidad } & \multicolumn{2}{|c|}{ Prudencia } & \multicolumn{2}{c|}{ Secreto } \\
\hline Categorías & $\mathbf{N}$ & $\mathbf{\%}$ & $\mathbf{N}$ & $\mathbf{\%}$ & $\mathbf{N}$ & $\boldsymbol{\%}$ & $\mathbf{N}$ & $\boldsymbol{\%}$ & $\mathbf{N}$ & $\boldsymbol{\%}$ \\
\hline Sí & 18 & 81,8 & 14 & 63,6 & 16 & 72,7 & 15 & 68,2 & 14 & 64 \\
\hline No & 4 & 18,2 & 8 & 36,4 & 6 & 27,3 & 7 & 31,8 & 8 & 36 \\
\hline
\end{tabular}

Fuente: datos obtenidos por los investigadores.

con un porcentaje del $100 \%$ se indica que es una formación integral, enmarcada en lo ético, moral y humanístico. Su actuar está enmarcado en principios y valores que le permiten ser sensible ante el dolor del otro y no perder su condición humana en la atención del paciente quirúrgico, razón de ser de este profesional (8).

En el presente estudio se encontró que un alto porcentaje de los instrumentadores quirúrgicos, frente a una situación en el ámbito quirúrgico, actuaría en coherencia con lo que se es, piensa y dice; asimismo, procedería de manera correcta, aunque esto afectara las relaciones personales, allí se ve aplicado el principio de veracidad; sin embargo, hay una minoría que actuaría en contra de ello, solo por no dañar su relación con los demás compañeros. Esto último invita a influir significativamente en todas las áreas del instrumentador quirúrgico, incluidos los aspectos éticos y bioéticos, ya que por la rutina, apatía y hasta desconocimiento se entra en un terreno donde se negocian los principios por agradar o complacer al resto del equipo quirúrgico, y por eso el reto de hoy es la actualización, no solo en los procedimientos quirúrgicos y equipos especializados, sino también en la formación del ser.

Entre las características recopiladas por las asociaciones de la profesión, al trabajar las competencias especí- ficas del instrumentador quirúrgico en Colombia se encuentran:

...la responsabilidad, tolerancia, democracia, respeto por la vida, vocación por el servicio y liderazgo, le permiten actuar interdisciplinariamente con el equipo de salud en el quirófano y fuera de él, mediante la aplicación de los procesos de instrumentación quirúrgica invasivos y no invasivos, esterilización, normas universales de bioseguridad, técnica aséptica y saneamiento ambiental (9).

Ante esto es importante destacar que el instrumentador quirúrgico, dentro de sus perfiles, puede afianzar los principios y valores de la ética, que lo llevan a respetar siempre a todo ser humano, por el simple hecho de ser un ser humano; sin embargo, uno de los obstáculos encontrados en la aplicación de estas características es la percepción personal de cada instrumentador quirúrgico ya que, al mirarlo de una manera objetiva, cada profesional es capacitado en principios y valores que lo llevarán a una mejor calidad en la atención, pero infortunadamente, algunos no convierten estos principios en vivencias para crecer a nivel personal y profesional; como señala Polo, "la ética no es para hacer cosas buenas sino para hacerse libremente bueno" (10). De tal manera, en el contexto quirúrgico el crecimiento 
personal influye en aspectos como la toma de decisiones en dilemas éticos que se presentan en el área de cirugía, ya que se necesita ejercer una autonomía personal más que acciones autónomas. Autores como Tom L. Beauchamp y James F. Childress marcan la diferencia entre autonomía personal y acciones autónomas, definiendo la primera como: "Regulación personal de uno mismo, libre, sin interferencias externas que puedan controlar, y sin limitaciones personales que impidan hacer una elección. Una persona actúa libremente de acuerdo con un plan elegido". Y la segunda "Las acciones autónomas se analizan en función de sus agentes, los cuales actuarán intencionadamente; con conocimiento y con ausencia de influencias externas que pretendan controlar y determinar el acto" (11). En relación con lo anterior, en esta investigación se evidencia que en la praxis del valor de la responsabilidad la mayoría de los encuestados reportó los eventos adversos que existieron durante un acto quirúrgico, lo importante del hallazgo es notar que estos profesionales tienen claro que se debe analizar, dar razón y asumir las consecuencias de las propias acciones u omisiones en lo referente al ejercicio de la instrumentación quirúrgica. Esto es valioso ya que, como decía Torralba, "la capacidad que tiene el ser humano de responsabilizar, no solo de sus acciones personales, sino del otro en tanto que ser frágil y vulnerable, pone de relieve su naturaleza ética" (12). Es decir, es notorio que la responsabilidad va más allá de cumplir con un horario y una función rutinaria; es vivir una vida enriquecida de valores morales enseñados desde el seno del hogar, reforzados en la academia universitaria, pero evidenciados cuando la tensión y el momento no sean del todo agradables; es ser un auténtico profesional con mayúscula cuando se admita un error, una negligencia en un acto operatorio.
Un profesional ha de poseer calidad técnica y calidad humana: no hay verdadera excelencia profesional en alguien carente de virtudes morales. Una persona corrompida puede ser "experta" en ciertos temas, hábil en una técnica, pero puede no ser un auténtico profesional, de él se espera no solo competencia técnica, sino también confianza y responsabilidad, con espíritu de servicio y calidad humana; en resumen, se le reclaman valores éticos (13)

En cuanto a la aplicación del principio de beneficencia en la labor del instrumentador quirúrgico, todas las decisiones deben buscar el máximo beneficio del paciente, el bien y el mejor interés en forma total, y no solamente la curación de la enfermedad; así, cada vez que una persona participante del equipo quirúrgico realice un acto, por muy sencillo que sea, debe buscar el bien del paciente, lo que implica pensar en el grado de beneficencia en que deben basarse las decisiones. Es preguntarse ¿si mi familiar estuviera allí qué haría yo, qué elemento suministrara? Por otro lado, la mitad de los instrumentadores quirúrgicos encuestados ha estado en desacuerdo con procedimientos que atentan contra la dignidad de los pacientes y la otra mitad no. En relación con este punto, el paciente debe tener un trato digno, por lo que es un ser valioso, único e irrepetible, dotado de un nombre y de una familia, con una trascendencia espiritual, y cuyas necesidades deben estar satisfechas. Asimismo, es importante hacer un esfuerzo por conocer y entender al paciente, especialmente los sujetos vulnerables (niños, adultos mayores, mujeres embarazadas, entre otros), Según Blázquez, "el bien del conjunto de la persona enferma debe predominar sobre el interés por el órgano enfermo y sus eventuales disfunciones" (14). También es necesario brindar un ambiente cálido y confortable. 
En resumen, se debe tener en cuenta lo propio del saber científico y actuar con profesionalismo y con un alto nivel ético.

\section{CONCLUSIÓN}

La Ley 1164, en su capítulo VI, establece parámetros generales que unifican los principios, valores, deberes y derechos del personal responsable que interviene y acompaña a los pacientes y hace referencia a la caracterización del talento humano en salud. Esta norma aporta y aclara la forma como se deben dar las relaciones entre personal de salud, paciente y familia, no solo en el ámbito clínico, sino también en el afectivo (amabilidad, comunicación).

Puesta en escena la percepción de principios y valores en los instrumentadores quirúrgicos encuestados se evidenció que la gran mayoría de ellos, en su quehacer profesional, aplican los principios y valores, aunque muchos desconocen el nombre o la definición de los mismos, entre ellos el mal menor, la no maleficencia, la totalidad y causa de doble efecto.

Los instrumentadores quirúrgicos respetan las creencias de los pacientes en relación con los procedimientos por realizar, e incluso intervienen cuando un paciente es discriminado, maltratado e instrumentalizado, esto es muy importante porque da evidencia de la integridad de su trabajo.

La mayoría de los instrumentadores tiene preferencias en la atención de determinados pacientes según sus caracteríasticas (niños, pacientes terminales o en estado crítico); estas, analizadas desde el punto de vista ético, son adecuadas y entrarían en el principio de beneficencia; sin embargo, hay un porcentaje mínimo pero atrayente que marca la preferencia cuando se trata de un familiar o un conocido; ante este grupo sería adecuado trabajar y reafirmar la escala de principios y valores profesionales, pues se sesga la atención y se viola el principio de la igualdad, el cual busca brindar el mismo cuidado a cada uno de los pacientes atendidos.

Se propone ahondar más, desde la academia, en el fortalecimiento de estos temas, ya que sería oportuno afirmar los principios y valores en el profesional de la salud para que este tenga una respuesta acorde con la demanda de atención de los pacientes, no solo en la resolución de los aspectos quirúrgicos, sino también en su estado emocional, trascendiendo a un nivel integral y de mayor cuidado, para que la atención oportuna y la comunicación personalizada no solo sean en el tiempo de la cirugía sino desde el pre y en el posquirúrgico.

Se debe insistir en el respeto y valor de la vida humana en todas sus dimensiones, con la necesidad de brindar un toque humanizado que traspase la barrera de ese atuendo quirúrgico y logre transmitir interés genuino por el paciente y respeto por su condición, para así seguir formando generaciones de profesionales con alto sentido ético y vocación, como "parte integral del equipo de salud" (15).

Conflicto de intereses: ninguno declarado.

\section{REFERENCIAS}

1. Gómez Pérez R. Problemas morales de la existencia humana. Madrid: Casals S.A; 1993.

2. Ministerio de Salud y Protección Social. Ley 1164 de 2007. 
3. The free dictionary. Gran Diccionario de la Lengua Española [visitado 2016 ago 1]. Disponible en: http:// es.thefreedictionary.com/principio

4. Mainetti JA. The Nuremberg Code. La Plata: Ética médica; 1947.

5. World Medical Association. Declaration of Helsinki. $64^{\mathrm{a}}$ Asamblea General. Fortaleza Brasil; 2013.

6. Comisión Nacional para la Protección de los Sujetos Humanos de Investigación Biomédica y del Comportamiento. Informe Belmont. USA; 1979.

7. Ministerio de Salud. Resolución 008430 de 1993. Normas Científicas, técnicas y administrativas para la investigación en salud. Santafé de Bogotá: Ministerio de Salud; 1993.

8. Naranjo Palomino NC, Delgado Arango NC. La instrumentación quirúrgica: caracterización y tendencias de la profesión en Colombia. Salud Areandina. 2012; 1(1): 116-36.

9. Asociación Colombiana de Instrumentadores Quirúrgicos Profesionales (Aciteq), Asociación Colombiana de Facultades de Instrumentación Quirúrgica (Acfiq), Colegio Colombiano de Instrumentación Quirúrgica (Coldisqui). Perfil y competencias del Profesional en Instrumentación Quirúrgica en Colombia; 2014.

10. Polo Barrera L. Ética: hacia una versión moderna de los temas clásicos. México: Publicaciones Cruz; 1993.

11. Bioetica web. Principio de autonomía y beneficiencia. Dos principios en tensión. 2008 [visitado 2016 ago 1]. Disponible en: http://www.bioeticaweb.com/autonomasa-y-beneficienciados-principios-en-tensiasn/.

12. Torralba Rosello F. Antropología del cuidar. Madrid: Mapfre; 1998.

13. Morales González JA, Napa Chapa G, Esquivel Soto J, Díaz Pérez LE. Principios de ética, bioética y conocimiento del hombre. México: Universidad Autónoma del Estado de Hidalgo; 2011.

14. Blázquez Fernández N. Bioética fundamental. Madrid: Biblioteca de Autores Cristianos; 1996.

15. Congreso de la República de Colombia. Ley 784 de 2002. Diario oficial 45.046. Colombia; 2002. 